Published in final edited form as:

J Urol. 2012 October ; 188(4): . doi:10.1016/j.juro.2012.06.019.

\title{
ENHANCING RENAL TUMORS IN PATIENTS WITH PRIOR NORMAL ABDOMINAL IMAGING: FURTHER INSIGHT INTO THE NATURAL HISTORY OF RENAL CELL CARCINOMA
}

\author{
Paul L Crispen ${ }^{1}$, Aldiana Soljic ${ }^{2}$, Gregory Stewart ${ }^{1}$, Alexander Kutikov ${ }^{3}$, Daniel Davenport ${ }^{1}$, \\ and Robert G Uzzo ${ }^{3}$ \\ ${ }^{1}$ University of Kentucky, Lexington, $\mathrm{KY}$ \\ 2University of Massachusetts, Wooster MA \\ ${ }^{3}$ Fox Chase Cancer Center, Philadelphia, PA
}

\section{Abstract}

Purpose: Patients undergoing serial cross sectional abdominal imaging to evaluate abdominal symptomatology may develop a renal tumor during their follow up of an unrelated disease process. Evaluation of such patients provides an opportunity to further define the radiographic inception, natural history and growth patterns of renal tumors.

Materials and Methods: A review of two institutions' renal tumor databases was performed for patients who developed an enhancing renal tumor with a prior normal cross sectional radiographic examination of the kidneys. Variables evaluated included: age, gender, tumor size at presentation, calculated tumor growth rate from negative scan to radiographic presentation and pathology in patients undergoing definitive treatment.

Results: We identified 36 patients with an average age of 65 yrs (range 44-82). Mean tumor size on presentation was $2.3 \mathrm{~cm}$ (range 1.0 -5.0). Presumed absolute growth rate based on timing of initial negative imaging study and tumor diameter at presentation was significantly greater compared to observed absolute growth rate following tumor detection $(0.71 \mathrm{~cm} / \mathrm{yr}$ versus 0.039 $\mathrm{cm} / \mathrm{yr}), \mathrm{p}=0.028$. No difference was noted between presumed and observed tumor growth based on absolute change in tumor volume $\left(1.44 \mathrm{~cm}^{3} / \mathrm{yr}\right.$ versus $\left.5.37 \mathrm{~cm}^{3} / \mathrm{yr}\right), \mathrm{p}=0.203$. Presumed relative growth rates based on tumor diameter $(665 \% / \mathrm{yr}$ versus $23 \% / \mathrm{yr})$ and volume $(1397 \% / \mathrm{yr}$ versus $169 \% / y r$ ) were significantly greater compared to observed relative growth rates, $\mathrm{p}=0.005$ and $\mathrm{p}=0.013$ respectively.

Conclusions: The presumed growth rate of the tumors was significantly greater than the observed growth rate suggesting that tumor growth rates do not follow a linear patter throughout their development and progression.

\section{Keywords}

renal tumors; active surveillance; natural history

Corresponding Author: Paul L. Crispen, MD Assistant Professor of Surgery 800 Rose Street, MS235 University of Kentucky Lexington, KY 40536 Crispen.paul@uky.edu Phone: 859-323-0487 Fax: 859-323-1944. 


\section{Introduction}

The routine use of cross-sectional abdominal imaging has lead to a significant increase in the detection of incidental renal tumors. The majority of these renal tumors are small, less than 4 centimeters in diameter, and undergo definitive therapy ${ }^{1}$. However, a small number of these tumors are not managed with definitive therapy and instead undergo active surveillance. The reasons patients undergo active surveillance are commonly based on other medical conditions rendering them suboptimal surgical candidates ${ }^{2}$.

Published series evaluating the active surveillance of renal tumors has provided valuable insight into their natural history. Based on available data, the majority of small renal tumors grow slowly with an average growth rate of approximately $3 \mathrm{~mm}$ per year ${ }^{3}$. Additionally, a significant proportion of renal tumors undergoing active surveillance will not demonstrate interval growth ${ }^{4}$. These findings suggest a rather indolent course for many small renal tumors, which is supported by the observed low rate of stage progression. Local tumor progression during active surveillance rarely influences future treatment options and is associated with pathologic upstaging in only $6 \%$ of cases $^{5}$. Furthermore, the rate of progression to metastatic disease appears to be low at approximately $2 \%$ over a median of approximately 3 years and is typically associated with rapid primary tumor growth during active surveillance ${ }^{6}$.

In an attempt to further our understanding of the natural history of renal tumors we evaluated a group of patients with prior normal renal imaging who subsequently developed enhancing renal tumors. Based upon the timing of prior normal imaging, we calculated the presumed growth rate of renal tumors and made comparisons to the observed growth rates of tumors following their clinical detection.

\section{Methods}

Following institutional review board approval, a retrospective review of two institutional renal mass databases was performed from 2005 to 2010 for patients who developed an enhancing renal mass following a prior normal contrast enhanced CT scan of the kidneys. During the study period the Fox Chase Cancer Center renal tumor database included 1,242 patients and the University of Kentucky renal tumor database included 590 patients. All lesions were locally confined to the kidney based upon standard radiographic staging protocols. Patients with hereditary renal cancer were excluded.

Variables examined included patient age, gender, indication for imaging, radiographic tumor features (cystic versus solid), tumor size (maximal diameter and volume), tumor growth (assumed and observed), and surgical pathology. Tumor size was measured as the maximal cross-sectional diameter. Tumor volume was calculated using the maximal cross sectional tumor diameter, using the equation $\mathrm{V}=0.523 \mathrm{x}^{3}$. Tumor growth was calculated as absolute and relative growth rates. Absolute tumor growth rates, based on tumor diameter (ATD) and volume (ATV), represent the change in tumor size per year. Relative tumor growth rates, based on tumor diameter (PTD) and volume (PTV), represent percentage change in tumor size per year. All growth rates were calculated as previously described ${ }^{2}$. Doubling time could not be used to evaluate changes in tumor volume because not all tumors demonstrated interval growth. The tumor diameter and volume for all lesions at the time of the normal CT scan was considered to be $0 \mathrm{~cm}$. Presumed growth was defined as the change in tumor size from the date of normal CT scan to the date of the CT scan demonstrating the renal tumor. Two presumed growth rates were calculated In order to establish a probable range of presumed growth rates, one based on tumor growth starting at the time of normal renal imaging and one based on tumor growth starting halfway between normal renal imaging and 
renal tumor detection. Presumed growth rate based on tumor growth starting at the time of normal renal imaging was utilized for all comparisons to observed growth rates. Observed growth was defined as the change in tumor size from the date of the first CT scan demonstrating a renal tumor to the date of the most recent CT scan. Observed tumor growth rates were calculated only in patients whose follow up after tumor detection was at least 12 months. In these patients, serial cross sectional imaging was performed at 3-6 month intervals and tumor size was measured at consistent levels within the kidney by direct comparison to prior studies. All CT scans, including the time zero scan, where the tumor was not identified, were reviewed by the treating Urologist.

Presumed versus observed growth rates were compared in individual patients using matched pairs non-parametric analyses (Wilcoxon Signed Rank Tests). SPSS Statistics software (IBM Corporation) version 19 was used to perform the analyses.

\section{Results}

We identified 36 patients meeting our inclusion criteria. Table 1 demonstrates patient demographics and radiographic tumor features at the time of the first CT demonstrating a renal tumor. The average time between the normal CT scan and the first CT scan demonstrating a renal tumor was 40.3 months (median 35, range 14-105). Figure 1 provides two examples of patients who developed a renal tumor following a prior normal radiographic examination. The indications for the initial CT scan demonstrating the renal tumor are presented in Table 1. The majority, 89\% (32/36), of patients did not have a history of renal cell carcinoma and were being evaluated for a number of other conditions. Symptoms (hematuria/flank pain) potentially attributable to a renal tumor were the indication for radiographic imaging in 25\% (9/36) patients. At the time of first detection, $94 \%$ (34/36) of tumors were less than $4 \mathrm{~cm}$ in maximal cross-sectional diameter and no patient had radiographic evidence of multifocal disease.

The average presumed ATD in all tumors was $0.81 \mathrm{~cm} / \mathrm{yr}$ (range $0.17-2 \mathrm{~cm} / \mathrm{yr}$ ) based on the interval between normal imaging and detection of renal tumor and $1.62 \mathrm{~cm} / \mathrm{yr}$ (range 0.34 -4.0 ) if tumor growth started half way between normal renal imaging and tumor detection. Figure 2 demonstrates presumed and observed tumor growth rates. Presumed and observed growth measurements for the 10 tumors with at least 12 months of observation after initial detection are presented in Table 2. Of the 10 patients undergoing at least 12 months of observation after initial detection, 20\% (2/10) did not demonstrate interval growth during surveillance. In comparing absolute presumed and observed growth rates, tumors demonstrated a significantly greater presumed growth rate compared to the observed growth rate based on tumor diameter, but not on tumor volume (Table 2). When comparing relative presumed and observed growth, tumors demonstrated significantly increased presumed growth rates based on tumor diameter and volume (Table 2).

In all tumors the average duration of follow up after the first positive study to treatment or the most recent CT scan in patients undergoing active surveillance was 10 months (median 3 , range $0-94)$. The majority of patients, $66 \%$ (22/36), underwent definitive management of their renal tumor. Malignant pathology was present in $82 \%$ (18/22) of treated tumors. Due to the majority of malignant renal tumors being clear cell renal cell carcinoma 83\% (15/18), the impact of tumor histology was not evaluated. In the 14 patients continuing active surveillance of their renal tumor, no metastatic progression has been noted during an average follow up of 9 months. 


\section{Discussion}

The active surveillance of renal tumors has provided tremendous insight into the natural history of renal cell carcinoma. Contemporary experience suggests that the majority of small renal tumors grow slowly and have a low rate of metastatic progression. Additionally, the data has provided an opportunity to study the growth kinetics of a solid human malignancy outside of an animal model. Tumor growth is believed to be influenced by multiple factors including the type and number of genetic mutations, blood supply, host immune status and tumor size. Concurrent and/or sequential genetic perturbations undoubtedly play a major role in a tumor's biologic potential; however, biomarkers predictive of a tumor's future growth or response to therapy have yet to be identified in renal cell carcinoma ${ }^{7,8}$. In regard to the relationship between tumor size and future tumor growth it is unclear if smaller or larger tumors grow faster. The theory of Gompertzian growth suggests that tumor growth prior to being clinically detectable is exponential and then slows once the tumor is larger and clinically detectable ${ }^{2}$. Here we have attempted to compare the growth rates of tumors prior to (presumed growth rate) and after (observed growth rate) clinical detection. When evaluating tumor growth based on change in diameter per year and relative growth in diameter and volume per year, the presumed growth rates were significantly greater than observed tumor growth. These results support the theory of Gompertizian growth in our small series of renal tumors.

Another clinical scenario which affords an opportunity to evaluate the presumed growth rate of renal tumors is the assessment of patients who develop metachronous real tumors during surveillance for previously treated RCC. In a review of 100 patients who developed a metachronous renal tumor in the contralateral kidney the median time to detection of the contralateral tumor was 5.8 years with an average size of $3 \mathrm{~cm}$, for a median presumed growth rate of $0.52 \mathrm{~cm} / \mathrm{year}^{9}$. This is similar to the median presumed growth rate in the current series of $0.56 \mathrm{~cm} / \mathrm{year}$. These presumed growth rates are greater than the majority of reported observed growth rates in prior series. In a report reviewing the experience of 8 single institutions including 234 patients, a median observed tumor growth rate of $0.28 \mathrm{~cm} /$ year was noted ${ }^{3}$. Additionally, in a recent report including 127 patients the observed growth rate was $0.13 \mathrm{~cm} / \mathrm{year}^{10}$. Taken together these findings suggest that the presumed growth rate may be in fact greater than the observed tumor growth rates. Although other series on the outcomes of metachronous renal tumors have been published, sufficient data is not presented to calculate presumed tumor growth rates ${ }^{11,12}$.

An additional way to evaluate the influence of tumor size on growth rates is to compare observed growth rates based upon tumor size at the time of presentation. It is often assumed that larger tumors grow faster than smaller tumors, reflecting a greater malignant potential. However, this assumption has challenged in most active surveillance series with no association between a tumor's growth and size at presentation ${ }^{3,13,14}$. There are several potential limitations of these observations, including the majority of tumors being less than 4 $\mathrm{cm}$ at the time of presentation, relatively short time of follow up, and comparisons based on linear growth rates opposed to volumetric growth. Most tumors, 86-87\%, included in the reported series are less than $4 \mathrm{~cm}$ in diameter (clinical stage $\mathrm{T} 1 \mathrm{a})^{3,14}$, thus not allowing accurate assessment of large tumor growth. Additionally most series are limited by a relatively short duration of follow up which could add further bias. These potential limitations are apparent in the report by Haramis et al who evaluated renal tumor growth in patients undergoing at least five years of active surveillance ${ }^{15}$. In this series of 44 patients with 51 renal tumors, the average tumor size on presentation was $2.7 \mathrm{~cm}$ and the average length of follow up was 6.4 years, which is nearly twice as long as prior series. The average observed growth rate of all tumors was $0.15 \mathrm{~cm} /$ year and was $0.31 \mathrm{~cm} /$ year in tumors greater than $4 \mathrm{~cm}$ at the time of presentation. The finding that tumors greater than $4 \mathrm{~cm}$ may exhibit 
faster growth rates is also suggested by Mues et al who examined the observed growth rate of renal tumors in a population of patients limited to having lesions greater than or equal to $4 \mathrm{~cm}$ at presentation ${ }^{16}$. In the 42 tumors that were included, the average tumor size at time of presentation was $7.1 \mathrm{~cm}$ and demonstrated an average growth rate of $0.57 \mathrm{~cm} /$ year. The results of these two studies suggest that additional follow up and inclusion of larger tumors may be necessary to elucidate the true impact of tumor size on future growth rates. In fact it may be that there is a temporal relationship between renal tumor growth rates with the impact of tumor size being more relevant at certain times during a tumor's progression with increased growth rates at the extremes of clinical detection.

Another potential bias when evaluating the impact of renal tumor growth size and tumor size is the manner in which growth rates are examined. In a previous study we have demonstrated that smaller tumors grow faster than larger tumors when considering proportional and volumetric growth ${ }^{2}$. This finding was also noted by Bratslavsky et al during the observation of renal tumors in patients with von Hippel-Lindau disease ${ }^{17}$. The discrepancy between these two studies and prior series can be explained simply by the method of measurements used to evaluate tumor growth. When evaluating volumetric and proportional growth, a similar change in diameter during a period of observation will have completely different meaning in a $1 \mathrm{~cm}$ versus $4 \mathrm{~cm}$ tumor. For example, a change in maximal diameter from $1 \mathrm{~cm}$ to $2 \mathrm{~cm}$ does not represent doubling the volume but rather a change from $0.52 \mathrm{~cm}^{3}$ to $4.19 \mathrm{~cm}^{3}$ or a $700 \%$ increase in tumor volume. However, a $1 \mathrm{~cm}$ increase in maximal diameter from $4 \mathrm{~cm}\left(33 \mathrm{~cm}^{3}\right)$ to $5 \mathrm{~cm}\left(65 \mathrm{~cm}^{3}\right)$ only represents a $97 \%$ change in tumor volume ${ }^{2}$. Another potential benefit of using tumor volume instead of tumor diameter is that tumor volume provides a better representation of changes in total tumor cell number ${ }^{2}$.

There are several limitations to the current manuscript that warrant mention including the small sample size and the manner of determining presumed tumor growth rates. The small sample size is based upon the inclusion of patients only with demonstrable normal imaging prior to detection of an enhancing renal tumor. With the continued routine use of cross sectional abdominal imaging, the number of patients fitting our inclusion criteria will likely increase as individual patients may undergo multiple radiographic evaluations throughout their lifetimes. Presumed tumor growth was calculated by setting the tumor size at zero at the time of normal renal imaging. Certainly the sensitivity of CT scans in detecting renal tumors less than a few millimeters needs to be considered and small clinically undetectable lesions may have been present on apparent normal renal imaging. Additionally, the CT protocols were not standardized with varying slice thickness and contrast phases between patients. However, the calculated presumed growth rates may also represent an underestimation of tumor growth as the exact time of growth initiation is unknown and may have occurred significantly after the negative scan. Additionally, observed growth rates are likely biased by only including tumors undergoing 12 months of observation, as tumors demonstrating rapid growth would be more likely to undergo definitive treatment. Despite these limitations the current series provides unique insight into the natural history of renal tumors.

\section{Conclusions}

Here we document patients with enhancing renal tumors which develop after a normal cross sectional renal imaging. This cohort represents a unique group which can provide insight into the natural history of small renal tumors. The presumed growth rate of the tumors was significantly greater than the observed growth rate of established tumors, suggesting that tumor growth kinetics vary over the tumor's lifetime. These findings support continued 
routine follow up of patients with enhancing renal tumors undergoing active surveillance even if a tumor as previously demonstrated slow growth.

\section{References}

1. Hollingsworth JM, Miller DC, Daignault S, et al. Rising incidence of small renal masses: a need to reassess treatment effect. J Natl Cancer Inst. 2006; 98:1331. [PubMed: 16985252]

2. Crispen PL, Viterbo R, Boorjian SA, et al. Natural history, growth kinetics, and outcomes of untreated clinically localized renal tumors under active surveillance. Cancer. 2009; 115:2844. [PubMed: 19402168]

3. Chawla SN, Crispen PL, Hanlon AL, et al. The natural history of observed enhancing renal masses: meta-analysis and review of the world literature. J Urol. 2006; 175:425. [PubMed: 16406965]

4. Kunkle DA, Crispen PL, Chen DY, et al. Enhancing renal masses with zero net growth during active surveillance. J Urol. 2007; 177:849. [PubMed: 17296355]

5. Crispen PL, Viterbo R, Fox EB, et al. Delayed intervention of sporadic renal masses undergoing active surveillance. Cancer. 2008; 112:1051. [PubMed: 18286513]

6. Smaldone MC, Kutikov A, Egleston BL, et al. Small renal masses progressing to metastases under active surveillance: A systematic review and pooled analysis. Cancer. 2011 Epub ahead of print.

7. Oosterwijk E, Rathmell WK, Junker K, et al. Basic research in kidney cancer. Eur Urol. 2011; 60:622. [PubMed: 21741760]

8. Banumathy G, Cairns P. Signaling pathways in renal cell carcinoma. Cancer Biol Ther. 2010; 10:658. [PubMed: 20814228]

9. Boorjian SA, Crispen PL, Lohse CM, et al. The impact of temporal presentation on clinical and pathological outcomes for patients with sporadic bilateral renal masses. Eur Urol. 2008; 54:855. [PubMed: 18487007]

10. Jewett MA, Mattar K, Basiuk J, et al. Active surveillance of small renal masses: progression patterns of early stage kidney cancer. Eur Urol. 2011; 60:39. [PubMed: 21477920]

11. Klatte T, Patard JJ, Wunderlich H, et al. Metachronous bilateral renal cell carcinoma: risk assessment, prognosis and relevance of the primary-free interval. J Urol. 2007; 177:2081. [PubMed: 17509291]

12. Rabbani F, Herr HW, Almahmeed T, et al. Temporal change in risk of metachronous contralateral renal cell carcinoma: influence of tumor characteristics and demographic factors. J Clin Oncol. 2002; 20:2370. [PubMed: 11981010]

13. Crispen PL, Wong YN, Greenberg RE, et al. Predicting growth of solid renal masses under active surveillance. Urol Oncol. 2008; 26:555. [PubMed: 18774473]

14. Rosales JC, Haramis G, Moreno J, et al. Active surveillance for renal cortical neoplasms. J Urol. 2010; 183:1698. [PubMed: 20299038]

15. Haramis G, Mues AC, Rosales JC, et al. Natural history of renal cortical neoplasms during active surveillance with follow-up longer than 5 years. Urology. 2011; 77:787. [PubMed: 21167561]

16. Mues AC, Haramis G, Badani K, et al. Active surveillance for larger (cT1bN0M0 and cT2N0M0) renal cortical neoplasms. Urology. 2010; 76:620. [PubMed: 20599256]

17. Bratslavsky, G.; Albert, P.; Liu, J., et al. Growth rates of hereditary clear cell carcinomas: influence of germline mutations and body mass index; Abstract presented at Society of Urologic Oncology Winter meeting; 2006. 


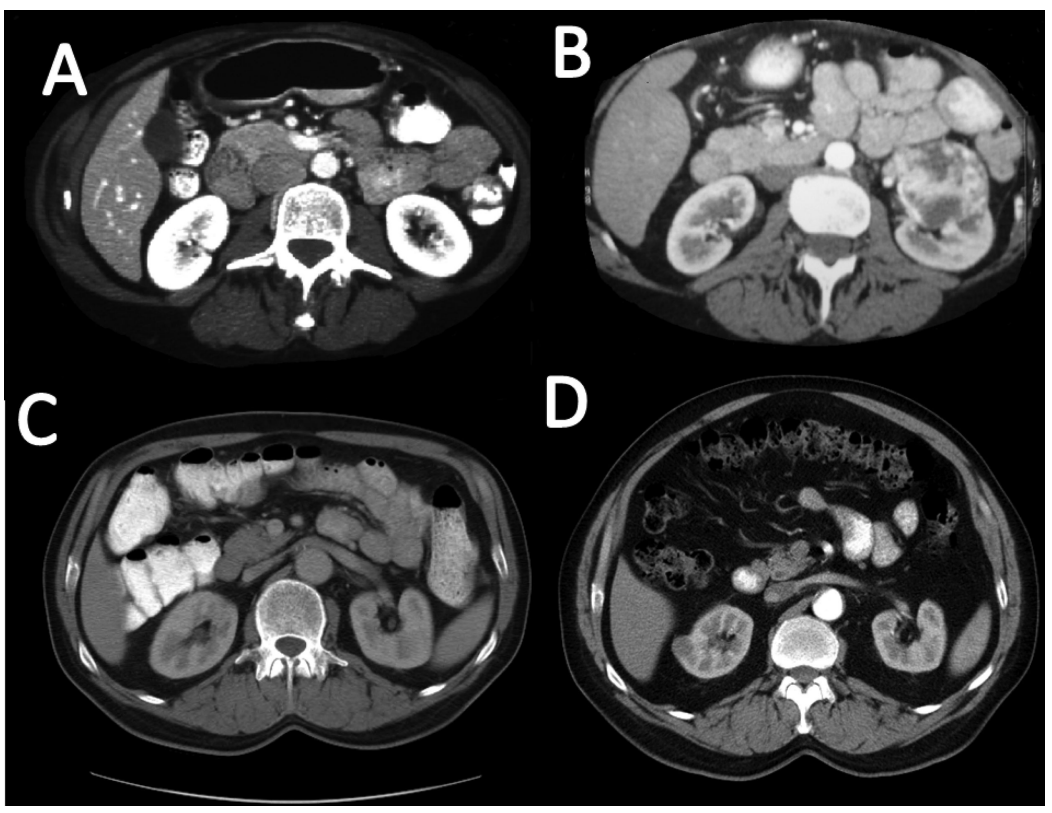

Figure 1.

CT scans of two patients demonstrating initial normal imaging without evidence of renal tumor and subsequent imaging demonstrating a renal tumor. Patient 1, Panel A represents normal renal imaging, Panel B shows $5 \mathrm{~cm}$ renal tumor on CT scan obtained 62 months after normal scan in the same patient as Panel A. Patient 2, Panel C represents normal renal imaging, Panel D shows $2.0 \mathrm{~cm}$ renal tumor obtained 69 months after normal scan in same patients as Panel C. 

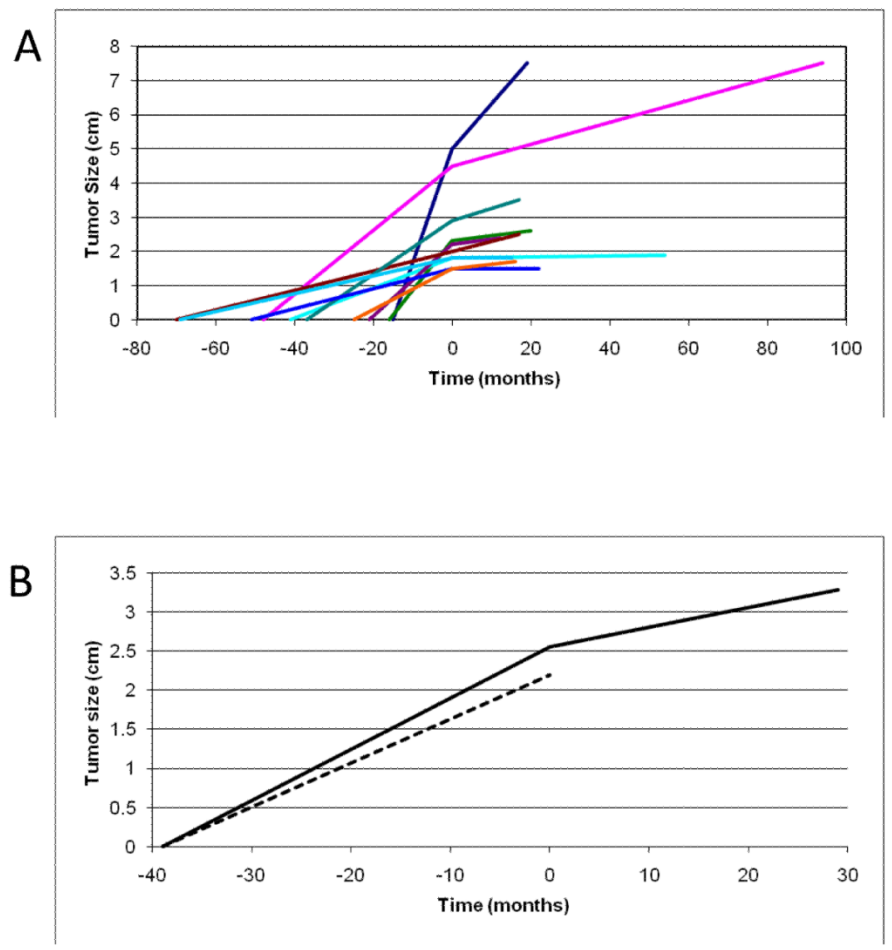

Figure 2.

Panel A shows linear presumed and observed growth rates of the 10 tumors with at least 12 months of observation after detection. Time 0 represents the time of first detection of the renal tumor. The slopes of the lines represent the absolute tumor growth rates. Panel B demonstrates the average absolute presumed and observed growth rates (solid lines). The dashed line represents the average absolute presumed growth rate of all 36 tumors. 
Table 1

\begin{tabular}{|c|c|}
\hline \multicolumn{2}{|c|}{ Patient Demographics } \\
\hline $\begin{array}{l}\text { Gender } \\
\text { Male } \\
\text { Female }\end{array}$ & $\begin{array}{l}20(56 \%) \\
16(44 \%)\end{array}$ \\
\hline Age (years) & $\begin{array}{c}\text { Average } 65 \\
\text { Median } 68 \\
\text { Range } 44-82\end{array}$ \\
\hline $\begin{array}{l}\text { Inication for CT scan } \\
\text { RCC follow up } \\
\text { Abdominal pain } \\
\text { Hematuria } \\
\text { Other abdominal disease }\end{array}$ & $\begin{array}{c}4(11 \%) \\
11(31 \%) \\
4(11 \%) \\
17(47 \%)\end{array}$ \\
\hline \multicolumn{2}{|c|}{ Radiographic Tumor Features at Preentation } \\
\hline Tumor Diameter (cm) & $\begin{array}{l}\text { Average } 2.3 \\
\text { Median } 1.9 \\
\text { Range 1-5 }\end{array}$ \\
\hline Tumor Volume $\left(\mathrm{cm}^{3}\right)$ & $\begin{array}{c}\text { Average } 11.8 \\
\text { Median 3.6 } \\
\text { Range 0.52-65 }\end{array}$ \\
\hline $\begin{array}{l}\text { Radiographic appearance } \\
\text { Solid } \\
\text { Cystic }\end{array}$ & $\begin{array}{c}30(83 \%) \\
6(17 \%)\end{array}$ \\
\hline
\end{tabular}


Table 2

Presumed and Observed Growth Rates

\begin{tabular}{|c|c|c|c|}
\hline & Presumed & Observed & p value * \\
\hline $\operatorname{ATD}(\mathbf{c m} / \mathbf{y r})$ & $\begin{array}{c}\text { Mean } 1.41 \\
\text { Median } 1.12 \\
\text { Range } 0.51-4.0\end{array}$ & $\begin{array}{c}\text { Mean } 0.39 \\
\text { Median } 0.28 \\
\text { Range } 0-1.58\end{array}$ & 0.005 \\
\hline $\operatorname{ATV}\left(\mathrm{cm}^{3} / \mathbf{y r}\right)$ & $\begin{array}{c}\text { Mean } 2.88 \\
\text { Mean } 1.08 \\
\text { Range } 0.61-13.08\end{array}$ & $\begin{array}{c}\text { Mean } 5.37 \\
\text { Median } 1.55 \\
\text { Range } 0-36.3\end{array}$ & 0.646 \\
\hline PTD $(\% / y r)$ & $\begin{array}{c}\text { Mean } 1331 \\
\text { Median } 1044 \\
\text { Range } 480-3840\end{array}$ & $\begin{array}{c}\text { Mean } 23 \\
\text { Median } 15 \\
\text { Range } 0-78\end{array}$ & 0.005 \\
\hline PTV (\%/yr) & $\begin{array}{c}\text { Mean } 2795 \\
\text { Median } 1017 \\
\text { Range } 571-12915\end{array}$ & $\begin{array}{c}\text { Mean } 169 \\
\text { Median } 56 \\
\text { Range } 0-670\end{array}$ & 0.007 \\
\hline
\end{tabular}

Wilcoxon signed rank tests. 